\title{
A Case of Intracranial Solitary Fibrous Tumor/Hemangiopericytoma Repeatedly Misdiagnosed as Hypertensive Intracerebral Hemorrhage
}

\author{
Ki Seong Eom ${ }^{1}$ (D), Hun-Soo Kim² \\ Departments of ${ }^{1}$ Neurosurgery, ${ }^{2}$ Pathology, Wonkwang University School of Medicine, Iksan, Korea
}

Received February 19, 2020

Revised May 6, 2020

Accepted May 7, 2020

\section{Correspondence}

Ki Seong Eom

Department of Neurosurgery,

Wonkwang University

School of Medicine,

895 Muwang-ro, Iksan 54538, Korea

Tel: +82-63-859-1462

Fax: +82-63-852-2606

E-mail: kseom@wonkwang.ac.kr
Because solitary fibrous tumor (SFT) and hemangiopericytoma (HPC) contain identical genetic abnormalities, the World Health Organization coined the term SFT/HPC to describe these lesions. Here, we present a rare case of SFT/HPC in a 40-year-old man with massive repeated intracranial hemorrhage $(\mathrm{ICH})$ in right medial temporal lobe. ICHs from a SFT/HPC were repeatedly misdiagnosed as hypertensive $\mathrm{ICH}$ by several departments and hospitals over approximately eight months, and the patient underwent inappropriate treatments. The amount of repeated $\mathrm{ICH}$ accompanying the tumor has increased significantly compared to the first $\mathrm{ICH}$, which may suggest rapid tumor growth. To avoid misdiagnosis and inappropriate treatment, surgeons should carefully examine all past and current patient-related radiological images and medical records before considering surgery.

Key Words Solitary fibrous tumor; Hemangiopericytoma; Misdiagnoses; Intracranial hemorrhages.

\section{INTRODUCTION}

Solitary fibrous tumor (SFT) is a rare spindle cell neoplasm. SFT in the central nervous system (CNS) was first described by Carneiro et al. [1] in 1996. Although SFT was originally thought to be primarily localized to the visceral pleura, it has subsequently been identified in almost all areas outside the thoracic region [2]. Hemangiopericytoma (HPC) is another rare, highly cellular vascularized mesenchymal tumor first described by Stout and Murray in 1942 [3]. HPC is caused by a malignant transformation of Zimmermann's pericytes, which surround the capillaries and postcapillary venules [3]. Although SFT is generally benign and can be completely removed, HPC have a high rate of recurrence and extracranial metastases such as bones, lungs, and liver [4,5]. The main treatment for HPC is radical resection, and postoperative radiation therapy is known to increase progression free survival [6]. Because SFT and HPC have been found to contain iden-

This is an Open Access article distributed under the terms of the Creative Commons Attribution Non-Commercial License (https://creativecommons.org/licenses/by-nc/4.0) which permits unrestricted non-commercial use, distribution, and reproduction in any medium, provided the original work is properly cited.

Copyright $\odot 2020$ The Korean Brain Tumor Society, The Korean Society for NeuroOncology, and The Korean Society for Pediatric Neuro-Oncology tical genetic abnormalities, the World Health Organization (WHO) coined the term SFT/HPC to describe these lesions in 2016 [7]. Intracranial hemorrhage (ICH) is a rare complication of SFT/HPC, and is associated with increased numbers and concentrations of blood vessels in the tumor, in addition to increased blood vessel fragility $[4,8]$. Here, we present a rare case of SFT/HPC that was repeatedly misdiagnosed as hypertensive ICHs by several departments and hospitals over approximately eight months. The repeated misdiagnosis occurred due to mistaken interpretations of the patient's radiological results.

\section{CASE REPORT}

On July 7, 2018, a 40-year-old man was admitted to our hospital after experiencing sudden weakness on his left side. Two years before presenting, the patient underwent a pacemaker insertion to correct for sick sinus syndrome. Eight months before presenting, the patient underwent burr-hole drainage (BHD) and extraventricular drainage surgery after being diagnosed with hypertensive ICH with intraventricular hemorrhage (IVH) at another hospital. At that time, preoperative and postoperative CT scans from another hospital (November 10, 
2017) showed huge ICH (size, $43 \times 36 \times 44 \mathrm{~mm}$ ) in the right medial temporal region with IVH in all ventricles (Fig. 1A, B). Postoperative CT scans showed the insertion of catheters through two burr holes into the region affected by $\mathrm{ICH}$ and the ventricle (Fig. 1C). The patient then received rehabilitative treatment and experienced no neurological deficits until presenting at our hospital. Upon presenting, a neurological examination revealed a slightly drowsy mental state (E3V5M6) and left hemiparesis (G4/4). A brain CT scan (July 7, 2018) revealed a very large area of acute $\mathrm{ICH}$ (size, $66 \times 72 \times 63 \mathrm{~mm}$ ) in the right temporal lobe with IVH (Fig. 2A, B). The patient was diagnosed with recurrent hypertensive ICH based on the similar location and nature of the patient's hypertensive ICH eight months before (Fig. 2C). The patient then underwent BHD surgery using two catheters. Two days after the operation, the patient's mental state had deteriorated to deep drowsy
(E3V4M6) and his left hemiparesis had also deteriorated to Grade 3. It is worth noting that unlike typical hypertensive $\mathrm{ICH}$, the patient's ICH could not be drained or aspirated through the catheters. A direct infusion of urokinase through the catheters two days after BHD surgery also did not result in any draining. Brain CT scans obtained three days (July 10, 2018) and five days (July 12,2018) after the BHD procedure revealed a slightly increased hematoma causing a severe mass effect (Fig. 3). Since the patient's clinical course and recent radiological findings were very different from those of typical hypertensive $\mathrm{ICH}$, the patient was referred to a neurosurgical oncology part. The patient's past radiological findings were reviewed again. Brain CT angiographies performed before the first BHD surgery at another hospital (November 10, 2017) and during the second BHD surgery at our hospital (July 7, 2018) showed a diffused blush in right medial temporal lobe, suggesting tu-
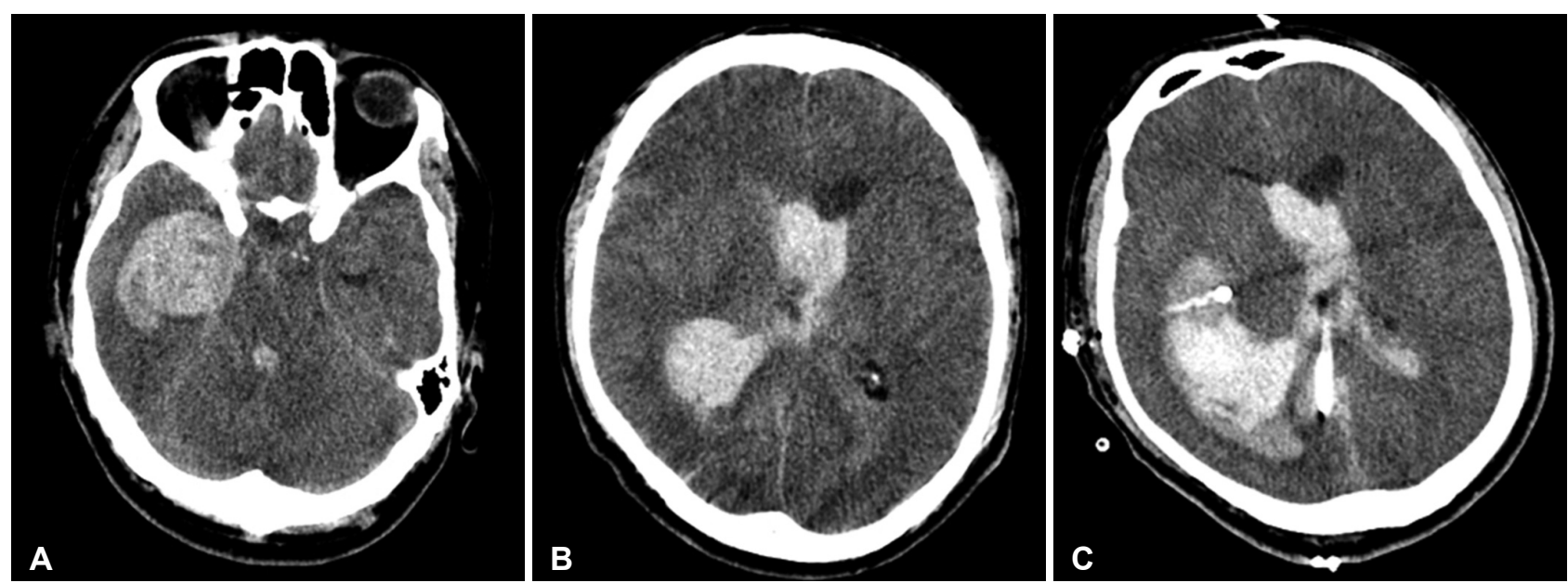

Fig. 1. Preoperative (A and $B$ ) and postoperative (C) CT images obtained from another hospital. Preoperative images show huge intracerebral hemorrhaging $(\mathrm{ICH})$ in the right medical temporal region with intraventricular hemorrhaging in all ventricles $(A$ and $B)$. The postoperative image shows insertion of catheters into the region affected by $\mathrm{ICH}$ and the ventricle through two burr holes (C).
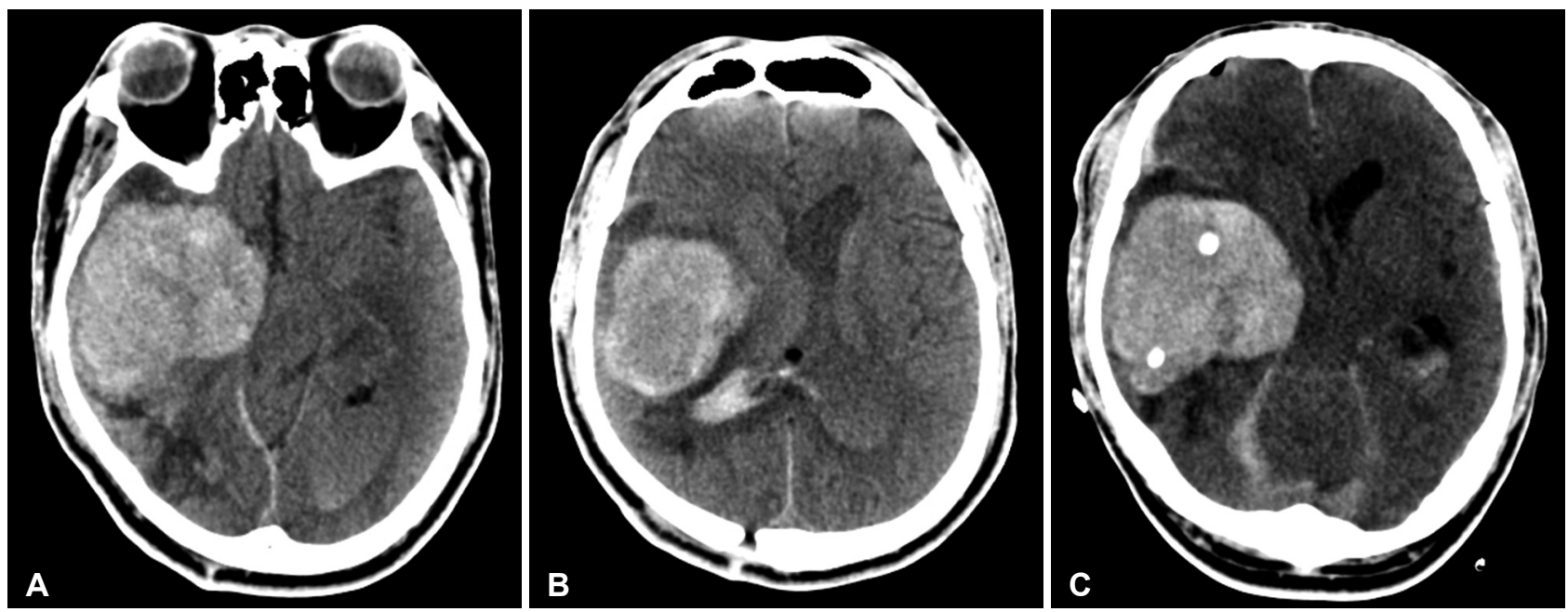

Fig. 2. Preoperative (A and $B$ ) and postoperative $(C) C T$ images obtained from our hospital. Images show a large area of acute intracerebral hemorrhaging $(\mathrm{ICH})$ in the right medial temporal lobe with intraventricular hemorrhaging $(A$ and $B)$ and the insertion of two catheters in to the region affected by $\mathrm{ICH}(\mathrm{C})$. 
mor staining (Fig. 4A, B). A brain CT scan from the Department of Rehabilitation Medicine (December 29, 2017) also revealed a solid mass lesion in the right medial temporal lobe (Fig. 4C). These finding had apparently been overlooked upon initial review. and the author concluded that the brain tumor had been misdiagnosed as hypertensive ICH. Brain MRI revealed a large, $75 \times 62 \times 57 \mathrm{~mm}$ acute hemorrhagic brain tumor in the right medial temporal lobe with heterogeneous diffuse enhancement. Perfusion MRI revealed increased relative cerebral blood volume in the right medial temporal lobe (July 11, 2018) (Fig. 5). Right carotid angiography revealed a large diffuse hypervascular tumor blush supplied from the menin- gohypophyseal and anterior choroidal arteries. Right extracarotid angiography revealed diffuse intense prolonged tumor staining supplied from the middle meningeal artery. Vertebral angiography revealed a diffuse tumor blush supplied from right cortical branch of the posterior cerebral artery. Six days after the second BHD procedure, the patient underwent a right frontotemporal craniotomy and gross total removal of the mass through the middle temporal gyrus. Microscopic examination of the tumor revealed perivascular cell proliferation, hypercellular sheets of monomorphous epithelioid cells, intratumoral staghorn vessels, pericellular reticulin staining, moderate nuclear atypia/cellularity, extensive hemorrhaging, and negative
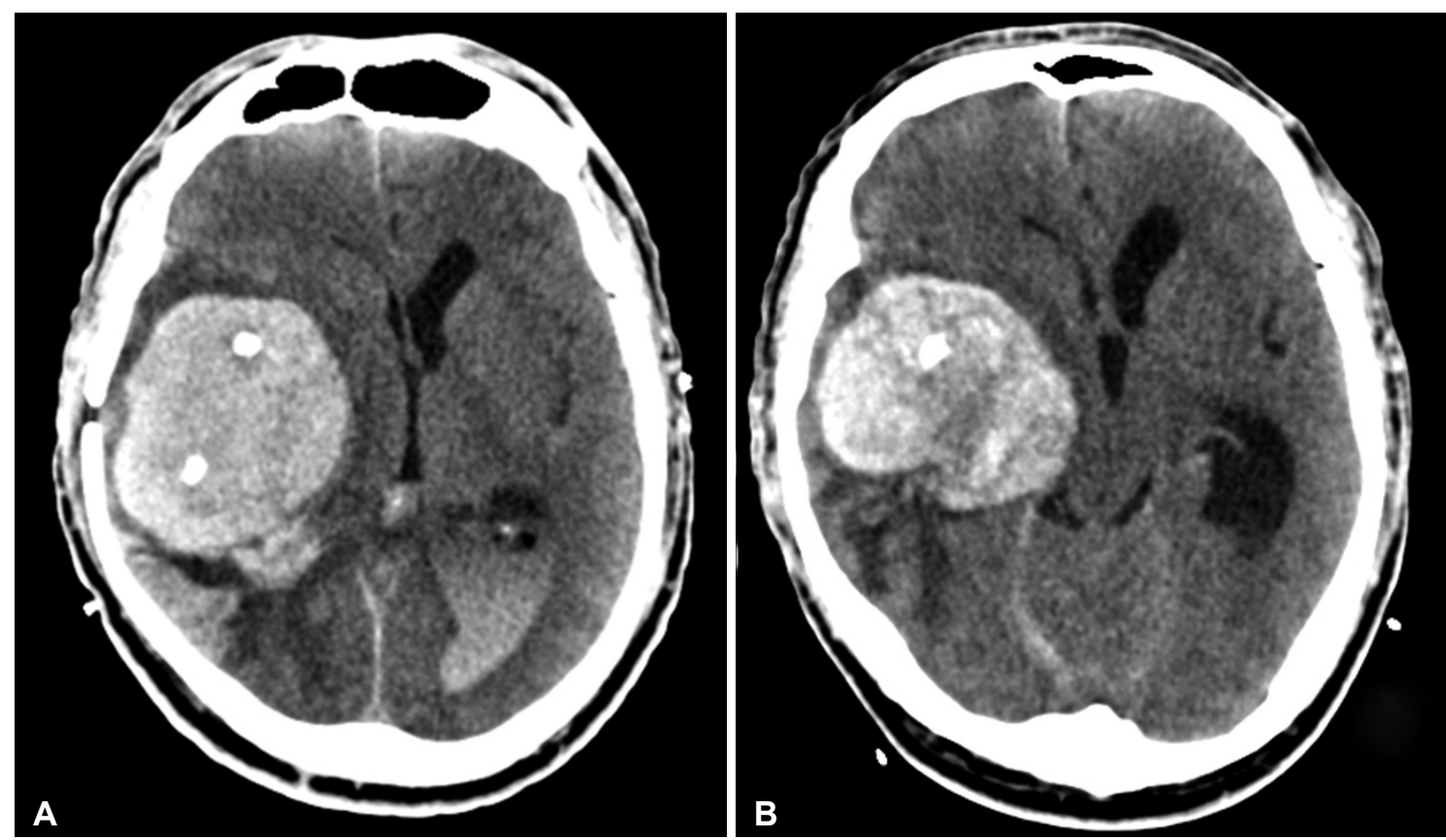

Fig. 3. Brain CT images obtained three days (A) and five days $(B)$ after burr-hole drainages at our hospital. Images show slightly increased hematoma causing a severe mass effect.
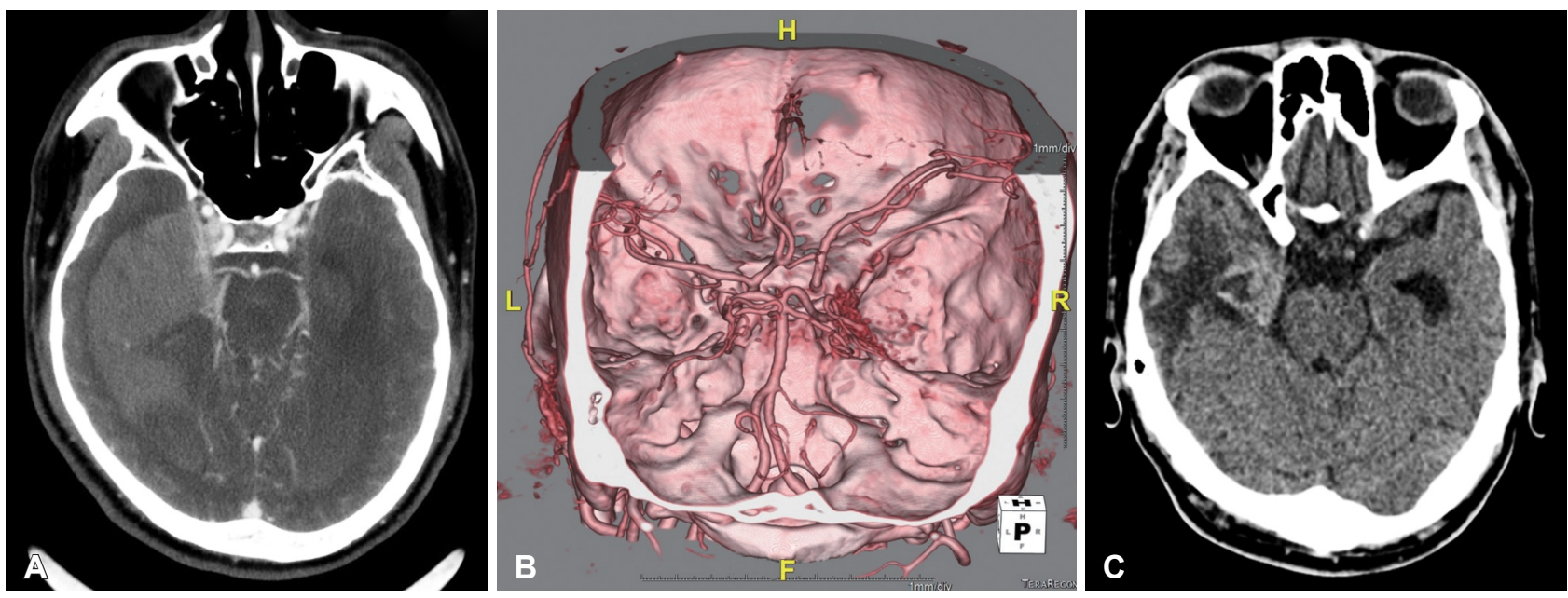

Fig. 4. Brain $C T$ angiographies performed before the first burr-hole drainages $(B H D)$ at another hospital $(A)$ and the second $B H D$ at our hospital (B). Images show diffused blush in the right medial temporal lobe, suggesting tumor staining. A brain CT scan from the Department of Rehabilitation Medicine $(\mathrm{C})$ also shows a solid mass lesion in the right medial temporal lobe. 
tumor necrosis (Fig. 6A-D). The mitotic index ranged from 10-12 mitoses/10 high power fields. Immunohistochemical finding showed negative for panCK, GFAP, S-100, CD34, CD99, vimentin, and AFP and pericelluar reticulin staining (Fig. 6E-H). The histological diagnosis was, therefore, SFT/ HPC (Grade III). The patient's postoperative course was uneventful, as he gradually recovered and his hemiparesis improved to Grade 4+. The study was approved by the Institute Ethical Committee in compliance with the institutional requirement (202002007).

\section{DISCUSSION}

SFT is characterized by small cells with dense or vesicular nuclei and scant cytoplasm individually separated by thin bands of collagen fiber [1,2]. Although SFTs in the CNS have generally been recognized as benign tumors (WHO Grade I tumors), their clinical behavior is unpredictable and sometimes they become malignant [9]. HPCs are known to undergo angiogenesis and develop pericytes around the capillaries. HPC cells also show more signs of mitosis and reticulin investment [3]. In the CNS, most HPCs are dural-based tumors that are located in the supratentorial area, falx, tentorium, dural sinus, and base of the skull [9]. HPCs can also be located in the lateral ventricle, pineal lesion, and intraparenchymal area $[10,11]$. Although the radiological characteristics of HPCs are similar to those of meningiomas, HPCs in the CNS are more prone to local recurrence and extracranial metastasis than meningiomas. HPCs also do not result in calcifications or hyperostosis of the skull as meningiomas commonly do. Ohba et al. [12] not-
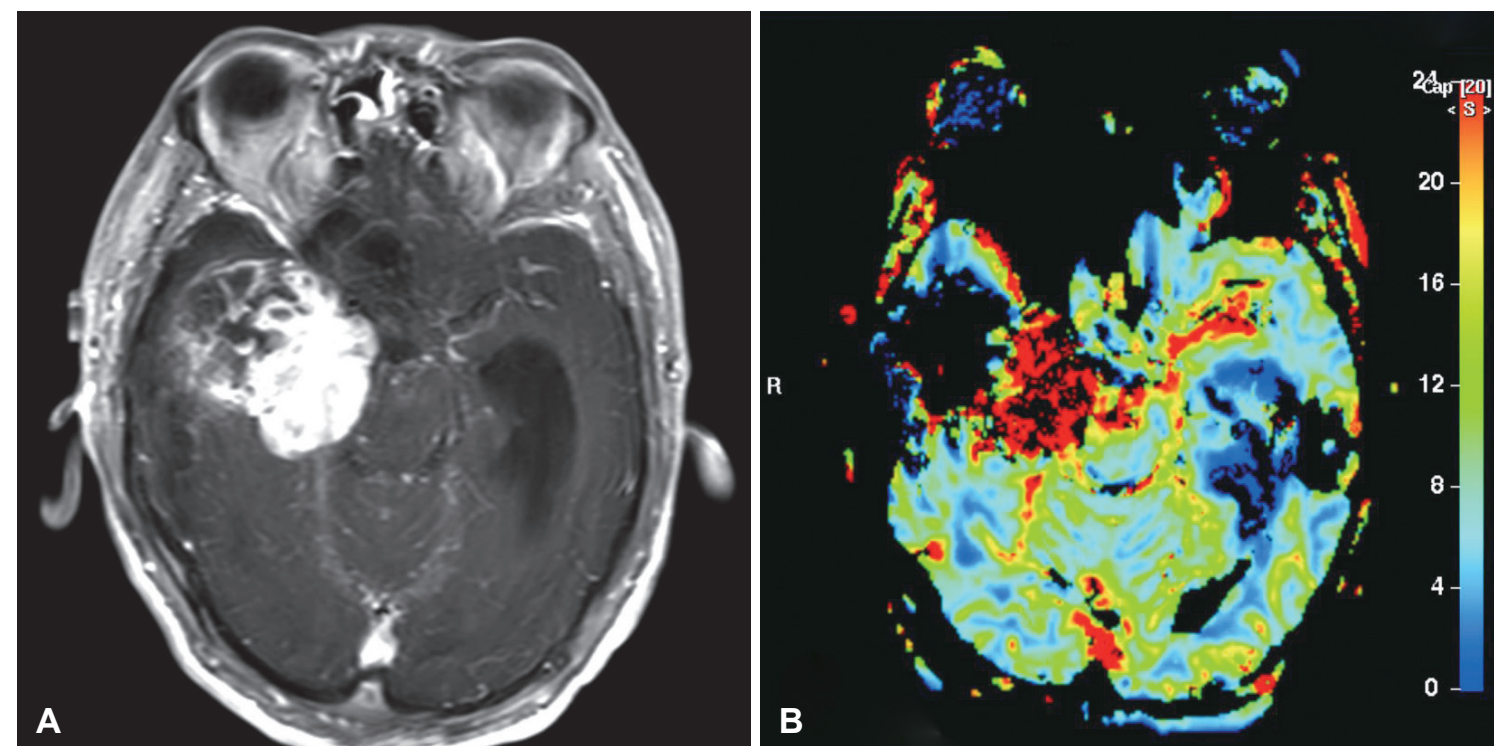

Fig. 5. Brain MRI shows a large, $75 \times 62 \times 57 \mathrm{~mm}$ acute hemorrhagic brain tumor in the right medial temporal lobe with heterogeneous diffuse enhancement (A). Perfusion MRI shows increased relative cerebral blood volume in the right medial temporal lobe (B).
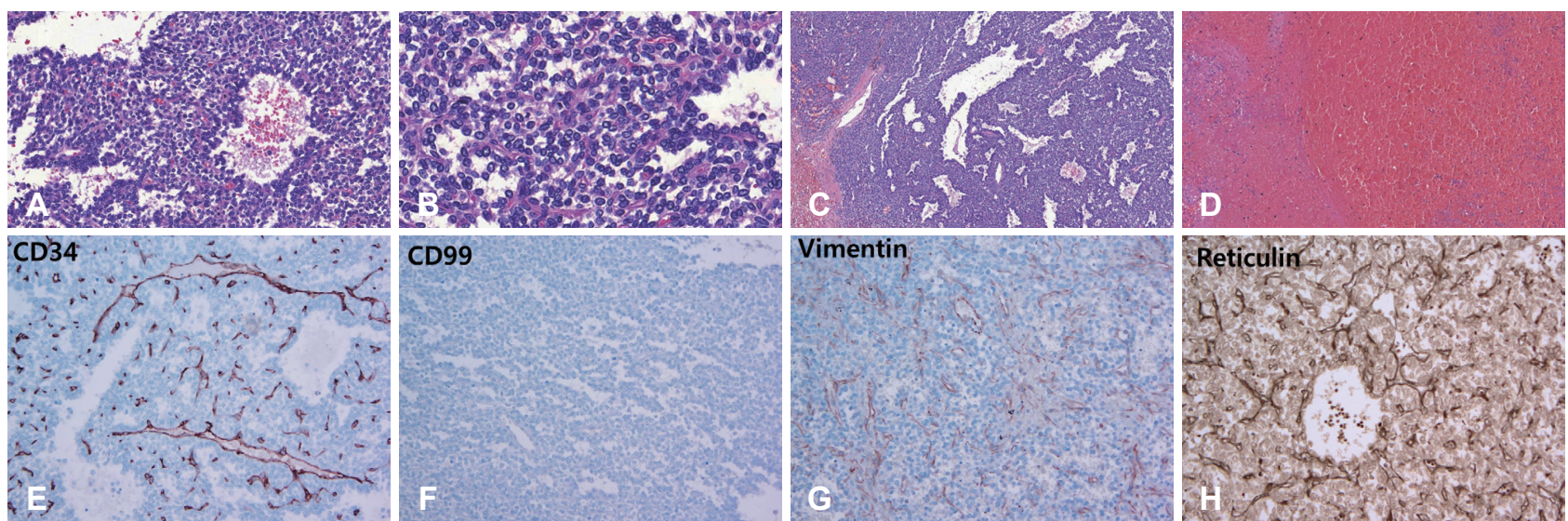

Fig. 6. Microscopic examinations of the tumor show perivascular cell proliferation, hypercelluar sheets of monomorphous epithelioid cells, intratumoral staghorn vessels, pericellular reticulin staining, moderate nuclear atypia and cellularity, and extensive hemorrhaging (H\&E; A: $\times 100, B$ : $\times 200$, C: $\times 40$, D: $\times 40)$. Immunohistochemical findings show negative for CD34, CD99, and vimentin (E: $\times 400, F: \times 100, G: \times 100)$ and pericellular reticulin staining $(\mathrm{H}: \times 400)$. 
ed that the age and myo-inositol levels calculated from magnetic resonance spectroscopy (MRS) are useful factors in distinguishing SFT/HPC from meningioma before surgery. They reported that there were significant differences between SFT/ HPC and meningioma in levels of glutamate, phosphocholine, myo-inositol, or glycerophosphocholine plus phosphocholine derived from long echo-time MRS, and myo-inositol derived from short echo-time MRS. They also mentioned that the age under 45 years and myo-inositol in short echo-time MRS of $\geq 6.347$ were associated with a diagnosis of SFT/HPC with high sensitivity and specificity [12].

The histological and immunohistochemical properties of intracranial SFTs and HPCs clearly overlap. Fritchie et al. [13] found the majority of soft-tissue SFTs and HPCs share inversions at 12q13, fusing the NAB2 and STAT6 genes. Therefore, SFTs and HPCs were integrated into a single disease entity in 2013 by the WHO Classification of Soft Tissue and Bone [14]. However, soft-tissue pathologists continued to diagnose such tumors as SFT, whereas neuropathologists retained the term HPC. Therefore, the combined term "SFT/HPC" was coined in the WHO's 2016 edition of the Classification of Tumors in the Central Nervous System [4,7]. This publication described three grades of SFT/HPC: 1) Grade I had been previously diagnosed as SFT, and corresponds most often to the highly collagenous spindle cell lesion with relatively low cellularity. 2) Grade II had been previously diagnosed in the CNS as HPC, and typically corresponds to a more cellular, less collagenous tumor containing plump cells and "staghorn" vasculature. 3) Grade III had been previously diagnosed as anaplastic HPC, and is diagnosed based on observing five or more mitoses per 10 high-power fields [7].

In 2018, Kim et al. [6] evaluated the clinical outcomes of SFT/HPC based on the 2016 WHO classification. They concluded that the recent WHO classification reflects the prognosis for both progression-free and overall survival better than the previous classification. SFT/HPC Grades II and III were associated with reduced progression times, and Grade III was significantly correlated with higher recurrence rates, more frequent extracranial metastases, and higher mortality rates. Kim et al. [6] further emphasized that the primary treatment goal for SFT/HPS should be gross-total resection of the tumor while preserving neurological function, and that radiotherapy can be administered as an adjuvant therapy. They also recommended that patients with Grade III SFT/HPC undergo long-term close surveillance for extracranial metastases, which is a common cause of death in these patients. In 2019, Sung et al. [9] also reported the results of treatment for SFT/ HPC based on the 2016 WHO classification. They concluded that patients with Grade I SFT/HPC experienced a more benign illness trajectory. Patients with Grade III SFT/HPC had a more aggressive illness trajectory and shorter overall survival compared to the Grade II group. The extent of both resection and adjuvant radiotherapy were important factors determining the rate of progression-free survival in the Grade II group. In the recurrence group, aggressive treatment was an important factor promoting patient survival. In addition, Sung et al. [9] recommended long-term follow-ups and periodic systemic evaluations to detect any delayed recurrence or systemic metastasis in SFT/HPC patients.

Most SFT/HPCs develop in dural tissue, and are often located in the base of the skull, the parasagittal sinus, and the falx cerebri [5]. Although the exact mechanism of such tumor bleeding is not known, histopathological studies reveal that $23.4 \%$ of HPCs display signs of microscopic intratumoral bleeding [11]. The hypothesized mechanisms of HPC bleeding include: fragile and immature blood vessels caused by tumor growth, staghorn-like vasculatures featuring changes in vascular distribution and structure, and vessel necrosis or occlusion due to endothelial proliferation [4]. Ha et al. [4] reported a case of acute massive hemorrhage caused by SFT/ HPC with high mitotic index. A high incomplete index indicates rapid tumor growth, potentially weakening immature blood vessels in the hypervascular tumor. They therefore hypothesized that this rapid tumor growth caused the acute hemorrhage. In this case, the patient was admitted to the hospitals for ICH that occurred twice (November 10, 2017 and July 7, 2018). Although repeated ICHs occurred at the same location (right medial temporal region), the second bleeding was much higher than the first. Even considering the artifacts caused by hemorrhage, the patient's MRI (July 11, 2018) showed a marked increase in tumor size compared to previous radiological findings. The mechanism of this marked increase in hematoma volume is assumed to be rapid tumor growth that results in injury of tumor vasculature or adjacent vessels of the brain.

In this case, two different hospitals and departments misdiagnosed SFT/HPC and provided inappropriate treatments to the patient. If doctors had more carefully checked the radiological images taken before and after the patient's operations, they could have seen signs indicating that the hemorrhaging was caused by the brain tumor. First, the patient's ICH was located in the right medial temporal lobe, and this location is generally not where hypertensive ICH occurs (November 10, 2017 and July 7, 2018). Second, brain CT angiographies in two hospitals showed a diffused blush in right medial temporal lobe (November 10, 2017 and July 7, 2018). Therefore, additional digital subtraction angiography should have been conducted to confirm whether this finding was tumor staining. Third, a brain CT scan of the Department of Rehabilitation Medicine performed after all hematomas disappeared showed solid mass lesions in the right medial temporal lobe and ad- 
ditional further evaluations such as MRI and MRS should have been performed to confirm the nature of brain tumor (December 29, 2017). However, all these radiological images suggesting brain tumors have been overlooked since the initial review, and the accurate diagnosis was delayed for eight months.

In conclusion, we present a rare case of SFT/HPC with repeated ICH. The amount of hematoma accompanying the tumor has increased significantly compared to the first ICH, which may suggest rapid tumor growth. This tumor was also repeatedly misdiagnosed as hypertensive ICH. To avoid misdiagnosis and inappropriate treatment, surgeons should carefully examine all past and current patient-related radiological images and medical records before considering surgery.

\section{Conflicts of Interest}

The authors have no potential conflicts of interest.

\section{ORCID iD}

Ki Seong Eom (D) https://orcid.org/0000-0002-8354-4024

\section{REFERENCES}

1. Carneiro SS, Scheithauer BW, Nascimento AG, Hirose T, Davis DH. Solitary fibrous tumor of the meninges: a lesion distinct from fibrous meningioma. A clinicopathologic and immunohistochemical study. Am J Clin Pathol 1996;106:217-24.

2. Thway K, Ng W, Noujaim J, Jones RL, Fisher C. The current status of solitary fibrous tumor: diagnostic features, variants, and genetics. Int J Surg Pathol 2016;24:281-92.

3. Stout AP, Murray MR. Hemangiopericytoma: a vascular tumor featuring Zimmermann's pericytes. Ann Surg 1942;116:26-33.
4. Ha MH, Jung TY, Kim SK, Lee KH, Kim D. Primary intraparenchymal central nervous system solitary fibrous tumor/hemangiopericytoma presenting with intracerebral hemorrhage: a case report. Brain Tumor Res Treat 2019;7:53-6.

5. Guthrie BL, Ebersold MJ, Scheithauer BW, Shaw EG. Meningeal hemangiopericytoma: histopathological features, treatment, and long-term follow-up of 44 cases. Neurosurgery 1989;25:514-22.

6. Kim BS, Kim Y, Kong DS, et al. Clinical outcomes of intracranial solitary fibrous tumor and hemangiopericytoma: analysis according to the 2016 WHO classification of central nervous system tumors. J Neurosurg 2018;129:1384-96.

7. Louis DN, Perry A, Reifenberger G, et al. The 2016 World Health Organization classification of tumors of the central nervous system: a summary. Acta Neuropathol 2016;131:803-20.

8. Shetty PM, Moiyadi AV, Sridhar E. Primary CNS hemangiopericytoma presenting as an intraparenchymal mass--case report and review of literature. Clin Neurol Neurosurg 2010;112:261-4.

9. Sung KS, Moon JH, Kim EH, et al. Solitary fibrous tumor/hemangiopericytoma: treatment results based on the 2016 WHO classification. J Neurosurg 2019;130:418-25.

10. Schiariti M, Goetz P, El-Maghraby H, Tailor J, Kitchen N. Hemangiopericytoma: long-term outcome revisited. Clinical article. J Neurosurg 2011;114:747-55.

11. Mena H, Ribas JL, Pezeshkpour GH, Cowan DN, Parisi JE. Hemangiopericytoma of the central nervous system: a review of 94 cases. Hum Pathol 1991;22:84-91.

12. Ohba S, Murayama K, Nishiyama Y, et al. Clinical and radiographic features for differentiating solitary fibrous tumor/hemangiopericytoma from meningioma. World Neurosurg 2019;130:e383-92.

13. Fritchie KJ, Jin L, Rubin BP, et al. NAB2-STAT6 gene fusion in meningeal hemangiopericytoma and solitary fibrous tumor. J Neuropathol Exp Neurol 2016;75:263-71.

14. Fletcher CDM, Bridge JA, Hogendoorn PCW, Mertens F. WHO classification of tumours of soft tissue and bone. 4th ed. Lyon: IARC Press; 2013. 